\title{
1
}

\section{Applications of Thin Orbits}

\author{
Alex Kontorovich (Rutgers University) ${ }^{1}$
}

\begin{abstract}
This text is based on a series of three expository lectures on a variety of topics related to "thin orbits," as delivered at Durham University's Easter School on "Dynamics and Analytic Number Theory" in April 2014. The first lecture reviews closed geodesics on the modular surface and the reduction theory of binary quadratic forms before discussing Duke's equidistribution theorem (for indefinite classes). The second lecture exposits three quite different but (it turns out) not unrelated problems, due to Einsiedler-Lindenstrauss-Michel-Venkatesh, McMullen, and Zaremba. The third lecture reformulates these in terms of the aforementioned thin orbits, and shows how all three would follow from a single "Local-Global" Conjecture of Bourgain and the author. We also describe some partial progress on the conjecture, which has lead to some results on the original problems.
\end{abstract}

\subsection{Lecture 1: Closed Geodesics, Binary Quadratic Forms, and Duke's Theorem}

This first lecture has three parts. In \$1.1.1, we review the geodesic flow on the hyperbolic plane to study closed geodesics on the modular surface. Then $\S 1.1 .2$ discusses Gauss's reduction theory of binary quadratic forms. Finally, in $\S 1.1 .3$, we combine the previous two discussions to

1110 Frelinghuysen Rd Piscataway, NJ 08854

email: alex.kontorovich@rutgers.edu

url: math.rutgers.edu/ alexk

The author is partially supported by an NSF CAREER grant DMS-1455705, an NSF FRG grant DMS-1463940, an Alfred P. Sloan Research Fellowship, and a BSF grant. 


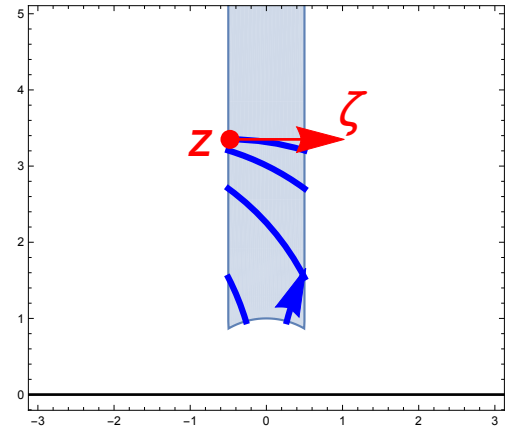

(a) As a broken ray

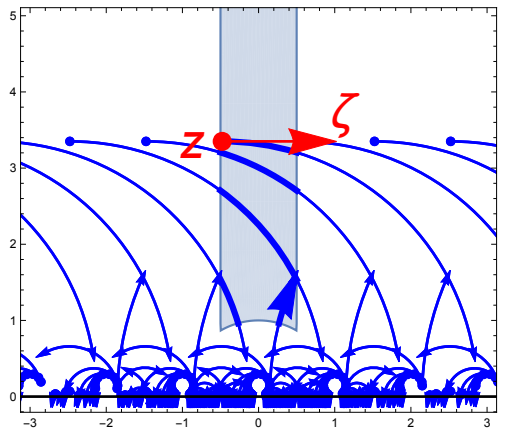

(b) As the collection of $\mathrm{PSL}_{2}(\mathbb{Z})$ translates

Figure 1.1 The geodesic flow on the modular surface

connect indefinite classes to closed geodesics, and state Duke's equidistribution theorem.

\subsubsection{Closed Geodesics}

Let $\mathbb{H}=\{x+i y: y>0\}$ denote the Poincaré (or perhaps more precisely, Beltrami) upper half plane, let $T \mathbb{H}$ be its tangent bundle, and for $(z, \zeta) \in$ $T \mathbb{H}$, equip the tangent bundle with Riemannian metric $\|\zeta\|_{z}:=|\zeta| / \Im z$. Here $z \in \mathbb{H}$ is the "position" and $\zeta \in T_{z} \mathbb{H} \cong \mathbb{C}$ is the "direction" vector. Let $T^{1} \mathbb{H}$ be the unit tangent bundle of all $(z, \zeta) \in T \mathbb{H}$ having $\|\zeta\|_{z}=1$. The fractional linear action of the group $G=\mathrm{PSL}_{2}(\mathbb{R})=\mathrm{SL}_{2}(\mathbb{R}) /\{ \pm I\}$ on $\mathbb{H}$ induces the following action on $T^{1} \mathbb{H}$ :

$$
G \ni\left(\begin{array}{ll}
a & b \\
c & d
\end{array}\right):(z, \zeta) \in T^{1} \mathbb{H} \mapsto\left(\frac{a z+b}{c z+d}, \frac{\zeta}{(c z+d)^{2}}\right),
$$

with invariant measure

$$
d \mu=\frac{d x d y d \theta}{y^{2}}
$$

in coordinates $(x+i y, \zeta)$, where $\arg \zeta=\theta$.

Exercise: This is indeed an action, which is moreover free, transitive, and invariant for the measure in (1.2).

The geodesics on $\mathbb{H}$ are vertical half-lines and semi-circles orthogonal to the real line. Given $(z, \zeta) \in T^{1} \mathbb{H}$, the time- $t$ geodesic flow moves $z$ along the geodesic determined by $\zeta$ to the point at distance $t$ from $z$. The visual point from some $(z, \zeta) \in T^{1} \mathbb{H}$ is the point on the boundary 
$\partial \mathbb{H} \cong \mathbb{R} \cup\{\infty\}$ that one obtains by following the geodesic flow for infinite time.

People studied such flows on manifolds purely geometrically for some time before Gelfand championed the injection of algebraic and representationtheoretic ideas. With Fomin [GF51], he discovered that under the identification

$$
G \cong T^{1} \mathbb{H}, \quad g \rightsquigarrow g(i, \uparrow),
$$

the geodesic flow on $T^{1} \mathbb{H}$ corresponds in $G$ to right multiplication by the diagonal subgroup

$$
A=\left\{a_{t}:=\left(\begin{array}{ll}
e^{t / 2} & \\
& e^{-t / 2}
\end{array}\right)\right\}
$$

see, e.g., [BM00, EW11].

To study (primitive, oriented) closed geodesics, we move to the modular surface, defined as the quotient $\Gamma \backslash \mathbb{H}$, with $\Gamma=\mathrm{PSL}_{2}(\mathbb{Z})$. Its unit tangent bundle $\mathcal{X}:=T^{1}(\Gamma \backslash \mathbb{H})$ is, as above, identified with $\Gamma \backslash G$, and the geodesic flow again corresponds to right multiplication by $a_{t}$. It is useful to think of this flow in two equivalent ways: (i) as a broken ray in a fundamental domain for $\mathcal{X}$ which is sent back inside when it tries to exit (see Figure 1.1a), or (ii) as a whole collection of $\Gamma$-translates of a single geodesic ray in the universal cover, $\mathbb{H}$, as in Figure 1.1b. Thus we will sometimes write $g \in \Gamma \backslash G$ for the first notion, and $\Gamma g$ for the second.

To obtain a closed geodesic on the modular surface, we start at some point $\Gamma g$ and come back to the same exact point (including the tangent vector) after a (least) time $\ell>0$ (here $\ell$ is for length). That is,

$$
\Gamma g a_{\ell}=\Gamma g, \quad \text { or equivalently, } \quad g a_{\ell}=M g,
$$

for some matrix ${ }^{2} M \in \Gamma$. Then

$$
M=g a_{\ell} g^{-1},
$$

so $M$ has eigenvalues $e^{ \pm \ell / 2}$, and $g$ is a matrix of eigenvectors. Note that $M$ is hyperbolic with trace

$$
\operatorname{tr} M=2 \cosh (\ell / 2),
$$

2 Technically, as $\Gamma=\mathrm{SL}_{2}(\mathbb{Z}) /\{ \pm I\}$, we should be using cosets $\pm M$ here. We will abuse notation and treat elements of $\Gamma$ as matrices, with the convention that their trace is positive. 
and the expanding eigenvalue, $\lambda$, say, is given by

$$
\lambda=e^{\ell / 2}=\left(\operatorname{tr} M+\sqrt{\operatorname{tr} M^{2}-4}\right) / 2 .
$$

Actually, since $\Gamma g$ is only determined up to left- $\Gamma$ action, the matrix $M$ is only determined up to $\Gamma$-conjugation (which of course leaves invariant its trace). In this way, primitive closed geodesics correspond to primitive (meaning not of the form $\left[M_{0}^{n}\right]$ for some $M_{0} \in \Gamma, n \geq 2$ ) hyperbolic conjugacy classes $[M]$ in $\Gamma$.

We note already from (1.5) that the lengths of closed geodesics are far from arbitrary; since $M \in \Gamma$, its eigenvalues are quadratic irrationals. It will also be useful later to note the visual point of $g$. Writing $M$ as $M=\left(\begin{array}{ll}a & b \\ c & d\end{array}\right)$, then if $c>0$, the matrix $g$ of eigenvectors can be given by Exercise:

$$
g=\frac{1}{\left(c^{2}\left(\operatorname{tr}^{2} M-4\right)\right)^{1 / 4}}\left(\begin{array}{cc}
\lambda-d & 1 / \lambda-d \\
c & c
\end{array}\right) .
$$

The scaling factor is to ensure $g$ has determinant 1 . If $c<0$, negate the first column in (1.6). Any other choice of $g$ is obtained by rescaling the first column by a factor $\sigma e^{t / 2}$ and the second by $\sigma e^{-t / 2}, \sigma \in\{ \pm 1\}$; this of course corresponds to the right action by $a_{t}$ in $\mathrm{PSL}_{2}(\mathbb{R})$. The visual point $\alpha$ from $g$ is determined by computing

$$
\alpha=\lim _{t \rightarrow \infty} g a_{t} \cdot i=\frac{\lambda-d}{c}=\frac{a-d+\sqrt{\operatorname{tr}^{2} M-4}}{2 c} .
$$

Note again that this is a quadratic irrational, and its Galois conjugate $\bar{\alpha}$ is the visual point of the backwards geodesic flow. Note also that $\alpha$ is independent of the choice of $g$ above. Finally, we record here that the fractional linear action of $M$ on $\mathbb{R}$ fixes $\alpha$; indeed, starting from $g a_{\ell}=M g$, multiply both side on the right by $a_{t}$, have that matrix act on the left by $i$, and take the limit as $t \rightarrow \infty$ :

$$
\alpha=\lim _{t \rightarrow \infty} g a_{\ell} a_{t} \cdot i=\lim _{t \rightarrow \infty} M g a_{t} \cdot i=M \alpha .
$$

To see an explicit example, let us construct the geodesic corresponding to the hyperbolic matrix

$$
M=\left(\begin{array}{cc}
12 & 5 \\
-5 & -2
\end{array}\right)
$$




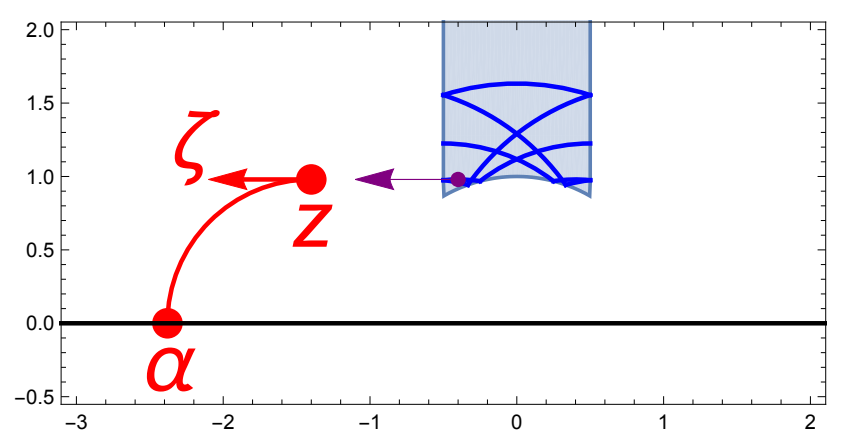

Figure 1.2 The closed geodesic corresponding to $M$ in (1.9)

From (1.5), (1.6), and (1.7), we compute

$\lambda=5+2 \sqrt{6}, \quad g=\frac{1}{\sqrt[4]{2400}}\left(\begin{array}{cc}-7-2 \sqrt{6} & 7-2 \sqrt{6} \\ 5 & -5\end{array}\right), \quad \alpha=\frac{-7-2 \sqrt{6}}{5}$.

Using the identification (1.3) and action (1.1), the point $g \in G$ corresponds to the point $(z, \zeta) \in T^{1} \mathbb{H}$ where

$$
z=-\frac{7}{5}+\frac{2 i \sqrt{6}}{5}, \quad \zeta=-\frac{2 \sqrt{6}}{5} .
$$

The points $z, \zeta$, and $\alpha$ are shown in Figure 1.2, as well as their images in the standard fundamental domain $\mathcal{F}$ for $\Gamma$. The resulting closed geodesic, also shown in $\mathcal{F}$, has length $\ell=2 \log \lambda \approx 4.58$. Had we started with $M^{2}$ instead of $M$, we would have obtained the same $g, z, \zeta$, and $\alpha$ but the length would double, corresponding to looping around the geodesic twice (hence our restriction to primitive geodesics and conjugacy classes). Replacing $M$ by some conjugate, $\gamma M \gamma^{-1}$, with $\gamma \in \Gamma$, results in replacing $g, z$, and $\alpha$ by $\gamma g, \gamma z$, and $\gamma \alpha$, respectively (of course the geodesic remains unchanged).

Next we wish to discuss the cutting sequence of the geodesic flow. Recall that $\Gamma$ is generated by two elements $T=\left(\begin{array}{ll}1 & 1 \\ 0 & 1\end{array}\right)$ and $S=\left(\begin{array}{cc}0 & 1 \\ -1 & 0\end{array}\right)$, and that its standard fundamental domain $\mathcal{F}$ is the intersection of the domains

$$
\mathcal{F}_{T}:=\{\Re z>-1 / 2\}, \mathcal{F}_{T^{-1}}:=\{\Re z<1 / 2\} \text {, and } \mathcal{F}_{S}:=\{|z|>1\} .
$$

As we follow the geodesic flow from $\mathcal{F}$, thought of as a subset of the universal cover, $\mathbb{H}$, we pass through one of the boundary walls, leaving one of the domains $\mathcal{F}_{L}, L \in\left\{T, T^{-1}, S\right\}$; here $L$ is the "letter" we must 

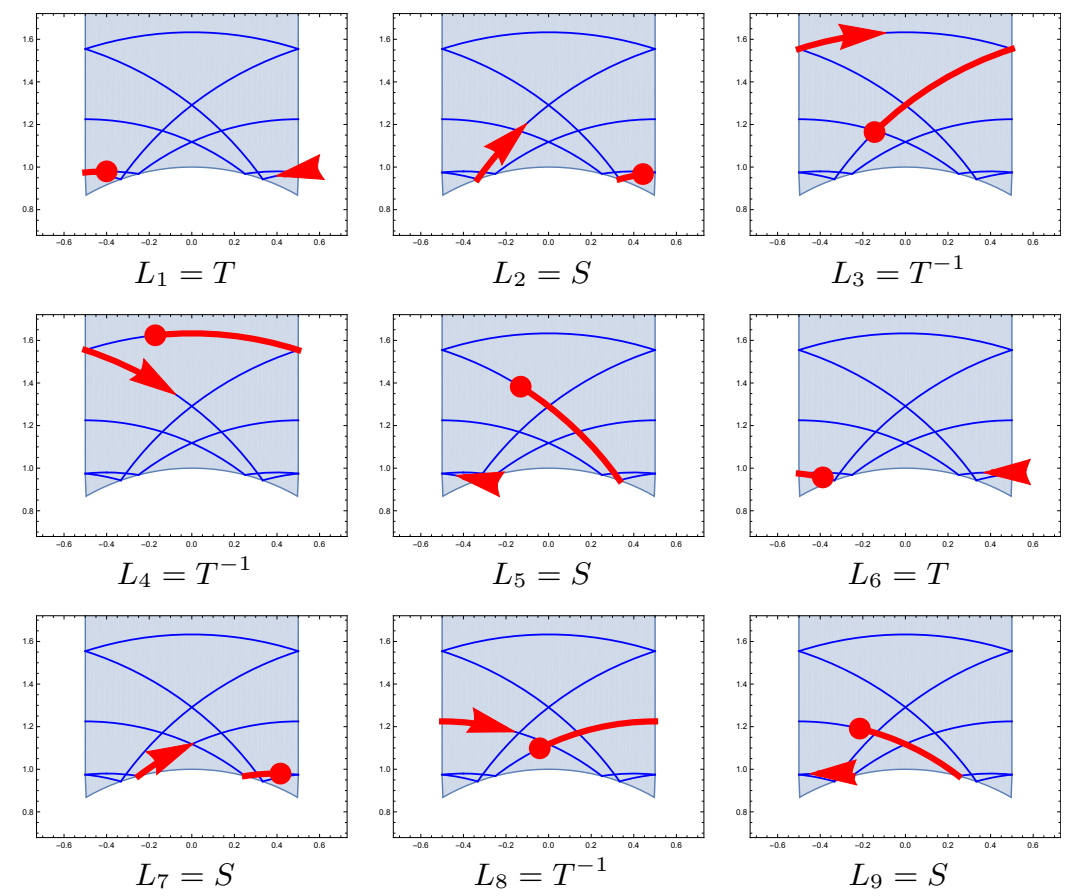

Figure 1.3 The cutting sequence of the geodesic flow

apply to return the flow to $\mathcal{F}$. Given a starting point $(z, \zeta) \in T^{1}(\Gamma \backslash \mathbb{H})$, its cutting sequence is this sequence of letters $L$.

To illustrate this, consider again the example in Figure 1.2. The flow first hits the wall $\Re z=-1 / 2$, and must be translated by $L_{1}=T$ back inside $\mathcal{F}$. Next the flow encounters the wall $|z|=1$, and is reflected using $L_{2}=S$. Continuing in this way (see Figure 1.3), we find that the cutting sequence of $(z, \zeta)$ in (1.11) is:

$$
T, S, T^{-1}, T^{-1}, S, T, S, T^{-1}, S, \ldots,
$$

repeating ad infinitum. It is easy to see from the geometry that such sequences are some number of $T$ 's or $T^{-1}$ 's separated by single $S$ 's. Computing these counts converts (1.12) into:

$$
\underbrace{T}_{1}, S, \underbrace{T^{-1}, T^{-1}}_{2}, S, \underbrace{T}_{1}, S, \underbrace{T^{-1}}_{1}, S, \ldots,
$$

which corresponds to the sequence

$$
1,2,1,1,1,2,1,1, \ldots
$$


repeating.

It seems to have first been observed by Humbert [Hum16] that this sequence should be compared to the continued fraction expansion of the visual point $\alpha$ of $(z, \zeta)$. We write the continued fraction expansion of any $x \in \mathbb{R}$ as

$$
x=a_{0}+\frac{1}{a_{1}+\frac{1}{a_{2}+\ddots}}=\left[a_{0}, a_{1}, a_{2}, \ldots\right],
$$

where $a_{0} \in \mathbb{Z}$ and the other $a_{j} \in \mathbb{N}$ are positive. These numbers are called the partial quotients of $x$, and we will sometimes call them "digits" or "letters." For the visual point $\alpha$ in (1.10), we compute:

$$
\alpha=\frac{-7-2 \sqrt{6}}{5}=[-3,1,1, \overline{1,1,1,2}],
$$

where the bar means repeating the last sequence of digits forever.

Comparing (1.14) to (1.13), we see that the periodic parts match, up to cyclic permutation (since anyway a closed geodesic has no canonical "starting" point). This leads us to the notion of a reduced form for $\alpha$.

Definition: A quadratic irrational $\alpha$ is called reduced if it and its Galois conjugate $\bar{\alpha}$ satisfy the inequalities:

$$
-1<\bar{\alpha}<0<1<\alpha .
$$

A representative $M$ of a hyperbolic conjugacy class $[M]$ is also called reduced if its visual point $\alpha$ is.

Exercise: A quadratic irrational $\alpha$ is reduced iff its continued fraction is exactly (as opposed to eventually) periodic.

How should we reduce the representative $M$ in (1.9)? It's actually quite easy. Note that, in general, if $\alpha$ has continued fraction expansion

$$
\alpha=\left[a_{0}, \ldots, a_{h}, \overline{a_{h+1}, \ldots, a_{h+\ell}}\right],
$$

then

$$
\left(\begin{array}{cc}
0 & 1 \\
1 & -a_{0}
\end{array}\right) \cdot \alpha=\frac{1}{\alpha-a_{0}}=\left[a_{1}, \ldots, a_{h}, \overline{a_{h+1}, \ldots, a_{h+\ell}}\right] .
$$

That is, such matrices eat away the first digit (this of course is the leftshift map from dynamics). For $\alpha$ in (1.14), we could try acting (on the left) by $\gamma_{0}=\left(\begin{array}{cc}0 & 1 \\ 1 & -1\end{array}\right) \cdot\left(\begin{array}{cc}0 & 1 \\ 1 & -1\end{array}\right) \cdot\left(\begin{array}{ll}0 & 1 \\ 1 & 3\end{array}\right)$ to make an exactly periodic continued fraction $[1,1,1,2]$. But this matrix $\gamma_{0}$ has determinant -1 , being an odd 
product of determinant -1 matrices. To act instead by an element of $\mathrm{PSL}_{2}$, we eat away one more digit, using the matrix

$$
\gamma=\left(\begin{array}{cc}
0 & 1 \\
1 & -1
\end{array}\right) \cdot\left(\begin{array}{cc}
0 & 1 \\
1 & -1
\end{array}\right) \cdot\left(\begin{array}{cc}
0 & 1 \\
1 & -1
\end{array}\right) \cdot\left(\begin{array}{cc}
0 & 1 \\
1 & 3
\end{array}\right)=\left(\begin{array}{cc}
2 & 5 \\
-3 & -7
\end{array}\right) \text {, }
$$

to obtain

$$
\widetilde{\alpha}=\gamma \alpha=\frac{1+\sqrt{6}}{2}=[\overline{1,1,2,1}] .
$$

That is, we replace $M$ by

$$
\widetilde{M}=\gamma M \gamma^{-1}=\left(\begin{array}{ll}
7 & 5 \\
4 & 3
\end{array}\right)
$$

with $\widetilde{M}$ now reduced.

In this way, the geodesic flow corresponds to the symbolic dynamics on the continued fraction expansion of the visual point of the flow. Actually, we have been quite sloppy; for example, it is not clear what one should do if the geodesic flow passes through an elliptic point of the orbifold $\Gamma \backslash \mathbb{H}$. There is in fact a better way of encoding the cutting sequence, as elucidated beautifully by Series [Ser85]. That said, the less precise (but more immediate) description given here will suffice for the purposes of our discussion below.

\subsubsection{Binary Quadratic Forms}

This will be a very quick introduction to an extremely well-studied and beautiful theory; see, e.g., [Cas78] for a classical treatment. The theory is largely due to Gauss (building on Lagrange and Legendre), as developed his 1801 magnum opus, Disquisitiones Arithmeticae.

For integers $A, B$, and $C$, let $Q=[A, B, C]$ denote the (integral) binary quadratic form $Q(x, y)=A x^{2}+B x y+C y^{2}$. The general problem being addressed was: Given $Q$, what numbers does it represent? That is, for which numbers $n \in \mathbb{Z}$ do there exist $x, y \in \mathbb{Z}$ so that $Q(x, y)=n$ ? The question is perhaps inspired by the famous resolution in the case $Q=x^{2}+y^{2}$ by Fermat (and other special cases due to Euler and others). See [Cox13] for a beautiful exposition of this problem. 
Some observations:

(i) If $A, B$, and $C$ have a factor in common, then so do all numbers represented by $Q$, and by dividing out this factor, we may and will assume henceforth that $Q$ is primitive, meaning $(A, B, C)=1$.

(ii) The set of numbers represented by $Q$ does not change if $Q$ is replaced by $Q^{\prime}(x, y)=Q(a x+b y, c x+d y)$, with $a d-b c= \pm 1$; this of course is nothing but an invertible (over integers!) linear change of variables.

Gauss defined such a pair of forms $Q, Q^{\prime}$ to be equivalent but for us it will be more convenient to use strict (some authors call this proper, or narrow) equivalence, meaning we only allow "orientation-preserving" transformations. That is, we will write $Q \sim Q^{\prime}$ only when there is some $\gamma \in \mathrm{SL}_{2}(\mathbb{Z})$ (as opposed to $\mathrm{GL}_{2}$ ) with $Q=Q^{\prime} \circ \gamma$.

Exercise: This is indeed an action, that is, $\left(Q \circ \gamma_{1}\right) \circ \gamma_{2}=Q \circ\left(\gamma_{1} \gamma_{2}\right)$, and hence $\sim$ is an equivalence relation.

For a given $Q$, the set of all $Q^{\prime} \sim Q$ is called a class (or equivalence class) and denoted $[Q]$. Because we are considering strict equivalence here, this is often called the narrow class of $Q$.

Exercise: If $Q \sim Q^{\prime}$ then $D_{Q}=D_{Q^{\prime}}$, where

$$
D_{Q}:=\operatorname{discr}(Q)=B^{2}-4 A C
$$

is the discriminant. That is, the discriminant is a class function (invariant under equivalence). Observe that discriminants are quadratic residues $(\bmod 4)$, and hence $D_{Q} \equiv 0$ or $1(\bmod 4)$.

Exercise: When $D<0$, the form $Q$ is definite, that is, it only takes either positive or negative values, but not both. When $D>0$, the form is indefinite, representing both positive and negative numbers.

If $\operatorname{discr}(Q)=D=0$, or more generally, if $D=D_{0}^{2}$ is a perfect square, then $Q$ is the product of two linear forms. Then the representation question is much less interesting, and will be left as an exercise. We exclude this case going forward.

Exercise: Let

$$
\alpha_{Q}=\frac{-B+\sqrt{D}}{2 A}
$$

be the root of $Q(x, 1)$ (assuming $A \neq 0$ ), and suppose $Q^{\prime}=Q \circ \gamma$. Then $\alpha_{Q^{\prime}}=\gamma^{-1} \cdot \alpha_{Q}$, where the action here of $\gamma^{-1}$ is by fractional linear transformations.

We have seen that if two forms are equivalent, then their discriminants 
agree. It is then natural to ponder about the converse: $\operatorname{Does} \operatorname{discr}(Q)=$ $\operatorname{discr}\left(Q^{\prime}\right)$ imply that $Q \sim Q^{\prime}$ ?

To study this question, let $\mathscr{C}_{D}$ be the set of all inequivalent, primitive classes having discriminant $D$,

$$
\mathscr{C}_{D}:=\{[Q]: \operatorname{discr}(Q)=D\},
$$

and let $h_{D}:=\left|\mathscr{C}_{D}\right|$ be its size; this is called the (narrow) class number.

This set $\mathscr{C}_{D}$ is now called the class group (it turns out there is a composition process under which $\mathscr{C}_{D}$ inherits the structure of an abelian group, but this fact will not be needed for our investigations; for us, $\mathscr{C}_{D}$ is just a set). If having the same discriminant implied equivalence, then all class numbers would be 1 . This turns out to be false, but actually it is not off by "very" much, in the following sense: the class number is always finite.

Theorem 1.1.1 (Gauss) For any non-square integer $D \equiv 0,1(4)$, we have:

$$
1 \leq h(D)<\infty .
$$

It is easy to see that $h(D) \geq 1$. Indeed, if $D \equiv 0(4)$, then $x^{2}-\frac{D}{4} y^{2}$ is primitive of discriminant $D$; and if $D \equiv 1(4)$, then $x^{2}+x y-\frac{D-1}{4} y^{2}$ works.

We give a very quick sketch of the very well-known Theorem 1.1.1, as it will be relevant to what follows. The proof decomposes according to whether or not the class is definite.

Sketch when $D<0$. Let $\alpha_{Q}$ be the root of $Q(x, 1)$ as in (1.21); since $Q$ is definite, $\alpha_{Q} \in \mathbb{H}$. We already discussed the standard fundamental domain $\mathcal{F}$ for $\Gamma \backslash \mathbb{H}$, so we know (from the exercise below (1.21)) that there is a transformation $\gamma \in \mathrm{SL}_{2}(\mathbb{Z})$ taking $\alpha_{Q}$ to $\mathcal{F}$. Such a transformation $\gamma$ is moreover unique (up to technicalities when $\alpha_{Q}$ is on the boundary of $\mathcal{F}$ ), and gives rise to a unique reduced representative $Q^{\prime}=Q \circ \gamma^{-1}$ in the class $[Q]$. It remains to show there are only finitely many such having a given discriminant $D<0$. This is an easy exercise using $\left|\Re\left(\alpha_{Q}\right)\right| \leq 1 / 2$ and $\left|\alpha_{Q}\right| \geq 1$; indeed, one finds that

$$
A \leq \sqrt{\frac{|D|}{3}}, \text { and }|B| \leq A,
$$

and hence the number of reduced forms is finite.

Example: Take $D=-5$. This $D$ is not congruent to 0 or $1 \bmod 4$, 
so instead we consider $D=-20$. Then $A \leq \sqrt{20 / 3}$, that is, $A$ is at most 2. If $A=1$, then $B=0$ or \pm 1 . The latter case, $B= \pm 1$, gives no integral solutions to $C=\left(B^{2}-D\right) /(4 A)$, but the former gives $C=5$, corresponding to the reduced form $Q_{0}=x^{2}+5 y^{2}$. Next we consider $A=2$, whence $B \in[-2,2]$. Only the choices $B= \pm 2$ give integral values for $C=3$, corresponding to forms $Q_{ \pm}=2 x^{2} \pm 2 x y+3 y^{2}$. Actually this turns out to be a boundary case, and the two forms $Q_{ \pm}$are equivalent. Hence the class group $\mathscr{C}_{-20}=\left\{Q_{0}, Q_{+}\right\}$has class number $h_{-20}=2$. The fact that this class number is not 1 is well known to be related to the failure of unique factorization in $\mathbb{Z}[\sqrt{-5}]$, e.g., the number 6 factors both as $6=2 \cdot 3$ and as $6=(1+\sqrt{5} i)(1-\sqrt{5} i)$.

Sketch when $D>0$. Again we assume $A>0$. This case is more subtle, as the root $\alpha_{Q}$ and its Galois conjugate are real, so cannot be moved to the fundamental domain $\mathcal{F}$. Instead we notice that, since $\alpha_{Q}$ is real and quadratic, it has an eventually periodic continued fraction expansion, and transformations $\gamma \cdot \alpha_{Q}$ simply add (or subtract) letters to (or from) this expansion. In particular, there is a $\gamma \in \mathrm{SL}_{2}(\mathbb{Z})$ which makes the continued fraction exactly periodic; that is, the transformed root $\alpha_{Q \cdot \gamma}$ then satisfies the familiar condition (1.15). (This is our first hint that indefinite forms are related to closed geodesics!) We thus call an indefinite form $Q$ reduced if its root $\alpha_{Q}$ is reduced. As before, it is easy to see using

$$
\alpha_{Q}=\frac{-B+\sqrt{D}}{2 A}>1, \quad-1<\bar{\alpha}_{Q}=\frac{-B-\sqrt{D}}{2 A}<0,
$$

that

$$
0<-B<\sqrt{D}, \quad \frac{1}{2}(\sqrt{D}+B)<A<\frac{1}{2}(\sqrt{D}-B),
$$

which forces the class number to be finite.

Example: Take $D=7$, or rather $D=28$, since $7 \not \equiv 0,1(4)$. Then the possibilities for $B$ range from -1 to -5 . To solve $B^{2}-D=4 A C$, we must have $B^{2}-D \equiv 0(4)$, which leaves only $B=-2$ or $B=-4$. In the former case, the possible positive divisors of $\left(B^{2}-D\right) / 4=-6$ are $A=1,2,3$, or 6 , of which only $A=2$ and 3 lie in the range $\frac{1}{2}(\sqrt{28}-2)<A<\frac{1}{2}(\sqrt{28}+2)$. These two give rise to the forms

$$
Q_{1}=2 x^{2}-2 x y-3 y^{2} \quad \text { and } \quad Q_{2}=3 x^{2}-2 x y-2 y^{2} .
$$

The latter case of $B=-4$ leads in a similar way to the two reduced forms $Q_{3}=x^{2}-4 x y-3 y^{2}$ and $Q_{4}=3 x^{2}-4 x y-y^{2}$. 
Note that, unlike the definite case, reduced forms are not unique in their class, as any even-length cyclic permutation of the continued fraction expansion of $\alpha_{Q}$ gives rise to another reduced form. So we cannot conclude from the above computation that $h_{28} \stackrel{?}{=} 4$. Writing $\alpha_{j}$ for the larger root of $Q_{j}, j=1, \ldots, 4$, we compute the continued fractions:

$$
\alpha_{1}=[\overline{1,1,4,1}], \alpha_{2}=[\overline{1,4,1,1}], \alpha_{3}=[\overline{4,1,1,1}], \alpha_{4}=[\overline{1,1,1,4}] .
$$

It is then easy to see by inspection that $Q_{1}$ and $Q_{3}$ are equivalent, as are $Q_{2}$ and $Q_{4}$, e.g.,

$$
Q_{1} \circ\left[\left(\begin{array}{cc}
0 & 1 \\
1 & -1
\end{array}\right)\left(\begin{array}{cc}
0 & 1 \\
1 & -1
\end{array}\right)\right]^{-1}=Q_{3} .
$$

(Exercise: Verify this and compute the change of basis matrix to go from $Q_{2}$ to $Q_{4}$.) Thus $h_{28}=2$.

Class groups and class numbers are extremely mysterious. A discriminant is defined to be fundamental if it is the discriminant of a (quadratic, in our context) field; such $D$ 's are either $\equiv 1(\bmod 4)$ and squarefree, or divisible by 4 with $D / 4$ squarefree and $\equiv 2,3(\bmod 4)$. Dirichlet's Class Number Formula (see, e.g., [Dav80, IK04]) gives an approach to studying class groups: If $D$ is a fundamental discriminant, then

$$
h_{D}=\sqrt{|D|} L\left(1, \chi_{D}\right) \times \begin{cases}1 /(2 \pi), & \text { if } D \leq-5, \text { or } \\ 1 / \log \epsilon_{D}, & \text { if } D>0 .\end{cases}
$$

Here $L\left(1, \chi_{D}\right)$ is called a "special $L$-value," and for $D>0$, the factor $\epsilon_{D} \in \mathbb{Q}(\sqrt{D})$ is determined by:

$$
\epsilon_{D}=\frac{t+s \sqrt{D}}{2},
$$

where $(T, S)=(t, s)$ is the least solution to the Pellian equation

$$
T^{2}-S^{2} D=4 .
$$

It follows from (1.26) that $\epsilon_{D}$ is a unit in $\mathbb{Q}(\sqrt{D})$, as its algebraic norm is $\epsilon_{D} \bar{\epsilon}_{D}=1$. (If the Pell equation (1.26) has no solutions with 4 replaced by -4 on the right side, then $\epsilon_{D}$ is the fundamental unit; otherwise it is the square of the latter.) The $L$-value, which we will not bother to define (as it is not relevant to our discussion), is so fascinating an object about which one could say so much, that we will instead say very little. For example, it is not hard to show that

$$
L\left(1, \chi_{D}\right) \leq C \log |D| .
$$


Siegel famously proved [Sie35, Lan35] the reverse inequality, that for any $\varepsilon>0$,

$$
L\left(1, \chi_{D}\right) \geq C_{\epsilon} \cdot|D|^{-\varepsilon},
$$

though the constant $C_{\varepsilon}$ is ineffective, that is, the proof does not give any means to estimate it for any given $\epsilon$. (These days, we have other, not as strong, but on the other hand, effective, estimates, see [Gol76, GZ86].) Either way, we may think of the $L$-value as very roughly being of size 1 . Then definite class groups are, very roughly, of size

$$
h_{-D} \approx \sqrt{|D|},
$$

while indefinite ones are of size

$$
h_{D} \approx \sqrt{D} / \log \epsilon_{D} .
$$

(We will not give the symbol $\approx$ a precise meaning here.) As a consequence, we obtain Gauss's conjecture, that for definite class numbers, $h_{-D} \rightarrow \infty$, as $-D \rightarrow-\infty$, see [Deu33, Hei34].

For indefinite forms, the behavior is even more mysterious, and it is a longstanding conjecture that infinitely often the class number is 1 :

$$
\liminf h_{D} \stackrel{?}{=} 1,
$$

where the limit is over fundamental $D \rightarrow+\infty$. (If one does not require fundamental discriminants, this problem was apparently solved long ago by Dirichlet, see [Lag80].) In light of (1.27), this conjecture suggests that the unit $\epsilon_{D}$, defined in (1.25), should infinitely often be massive, of size about $e^{\sqrt{D}}$. Today no methods are known to force such large solutions to the Pell equation (1.26), despite rather convincing evidence (see, e.g., [CL84]) that this event is far from rare. On the other hand, it is quite easy to make giant class numbers, since one can force very small units, e.g., by taking $D$ 's of the form $D=t^{2}-4$, one sees that $\epsilon_{D}=\frac{t+\sqrt{D}}{2} \approx \sqrt{D}$, and $h_{D} \approx \sqrt{D} / \log D$ is as large as possible. Another long-standing conjecture in the indefinite case is that the average class number is roughly bounded, in the crude (more refined conjectures are available) sense that:

$$
\sum_{0<D<X} h_{D} \stackrel{?}{=} X^{1+o(1)} .
$$

We have insufficient space to delve further into this fascinating story, so leave it there. 


\subsubsection{Duke's Theorem}

We now combine the previous two sections, giving a bijection between primitive, indefinite classes $[Q]$ and primitive, oriented, closed geodesics $\gamma$ on the modular surface. This equivalence was apparently first observed by Fricke and Klein [FK90], and is also discussed in many places, e.g., [Cas78, Sar80, Hej83, IK04, Sar07].

We first attach a form $Q$ to a given hyperbolic matrix $M=\left(\begin{array}{ll}a & b \\ c & d\end{array}\right)$, the passage being through equating $\alpha$ 's in (1.7) and (1.21), as follows. Pattern matching (1.7) with (1.21), we obtain a preliminary (possibly imprimitive) set of variables $B_{0}=d-a, A_{0}=c, D_{0}=\operatorname{tr}^{2} M-4$, leading to

$$
C_{0}=\frac{B_{0}^{2}-D_{0}}{4 A_{0}}=-b .
$$

Setting $s=\operatorname{gcd}\left(A_{0}, B_{0}, C_{0}\right)$, the primitive quadratic form $\left[A_{0}, B_{0}, C_{0}\right] / s$ is almost what we want, but does not quite work, because $M$ is really in $\mathrm{PSL}_{2}(\mathbb{Z})$, and as is, $-M$ could give a different form. To fix this, we set

$$
Q=\frac{\operatorname{sgn}(\operatorname{tr} M)}{s}[c, d-a,-b],
$$

which is now well defined on $\mathrm{PSL}_{2}(\mathbb{Z})$. The discriminant of this form is

$$
D=\frac{\operatorname{tr}^{2} M-4}{\operatorname{gcd}(c, d-a, b)^{2}} .
$$

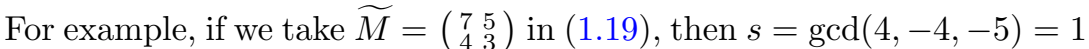
and $\operatorname{sgn}(\operatorname{tr} M)=+1$, so $\widetilde{M}$ corresponds to the binary quadratic form $\widetilde{Q}=4 x^{2}-4 x y-5 y^{2}$ of discriminant 96 . Note that if we had done the same operation starting with $M$ in (1.9), the form $Q=-5 x^{2}-14 x y-5 y^{2}$, also of discriminant 96 , and of course, $Q=\widetilde{Q} \circ \gamma^{-1}$ with change of variables matrix $\gamma$ given by (1.17). (Exercise: Verify all this.)

To invert the map (1.30) given some $Q=[A, B, C]$ with discriminant $D>0$ and not a perfect square, we seek a matrix $M=\left(\begin{array}{cc}a & -C s \\ A s & d\end{array}\right) \in$ $\mathrm{SL}_{2}(\mathbb{Z})$ so that $a+d>0$, say, and $d-a=B s$. Inserting the last identity into the determinant equation and completing the square gives:

$$
1=a^{2}+B s a+A C s^{2}=\frac{1}{4}(2 a+B s)^{2}-\frac{1}{4} D s^{2} .
$$

Multiplying both sides by 4 , we come to the familiar Pellian equation $(1.26)$; if $(t, s)$ is a fundamental solution, then $a=(t-B s) / 2, d=$ 
$(t+B s) / 2$, and we have found our desired hyperbolic matrix

$$
M=\left(\begin{array}{cc}
(t-B s) / 2 & -C s \\
A s & (t+B s) / 2
\end{array}\right) .
$$

That $M$ is primitive follows from the fundamentality of the solution $(t, s)$ (Exercise). For example, to turn $Q_{1}=2 x^{2}-2 x y-3 y^{2}$ of discriminant $D=28$ into a closed geodesic, we find the fundamental solution $(t, s)=$ $(16,3)$ to $(1.26)$, leading to $M_{1}=\left(\begin{array}{cc}11 & 9 \\ 6 & 5\end{array}\right)$.

Note that the key to finding $M$ above is to solve a Pellian equation, which itself goes through continued fractions; here is a more direct version of this inverse map. We first state the following simple

Exercise: The matrix

$$
M=\left(\begin{array}{cc}
a_{0} & 1 \\
1 & 0
\end{array}\right) \cdot\left(\begin{array}{cc}
a_{1} & 1 \\
1 & 0
\end{array}\right) \cdots\left(\begin{array}{cc}
a_{\ell} & 1 \\
1 & 0
\end{array}\right)
$$

fixes the real quadratic irrational $\alpha$ having continued fraction expansion $\alpha=\left[\overline{a_{0}, a_{1}, \ldots, a_{\ell}}\right]$. [Hint: Compare to (1.16).]

Recall from (1.8) that the desired matrix $M_{1}$ fixes $\alpha_{1}$, where $\alpha_{1}=$ $(2+\sqrt{28}) / 4$ is the root of $Q_{1}(x, 1)$. As the continued fraction expansion of $\alpha_{1}$ is given in (1.23), it is trivial to find the corresponding matrix $M_{1}$ :

$$
M_{1}=\left(\begin{array}{ll}
1 & 1 \\
1 & 0
\end{array}\right) \cdot\left(\begin{array}{ll}
1 & 1 \\
1 & 0
\end{array}\right) \cdot\left(\begin{array}{ll}
4 & 1 \\
1 & 0
\end{array}\right) \cdot\left(\begin{array}{ll}
1 & 1 \\
1 & 0
\end{array}\right)=\left(\begin{array}{cc}
11 & 9 \\
6 & 5
\end{array}\right),
$$

again. If the product in (1.32) had odd length, one would obtain a matrix of determinant -1 , whose square is the desired element of $\mathrm{SL}_{2}(\mathbb{Z})$. This corresponds to the situation when $T^{2}-D s^{2}=-4$ is solvable; then, writing $(t, s)$ for the least solution, we find that $\epsilon_{D}$ is expressed as $\eta_{D}^{2}=\epsilon_{D}$, where $\eta_{D}=(t+s \sqrt{D}) / 2$ is the fundamental unit.

Definition: The discriminant of a closed geodesic $\gamma$ on the modular surface, or its corresponding hyperbolic conjugacy class, is defined to be that of its associated equivalence class of binary quadratic forms. Explicitly, the discriminant of $M=\left(\begin{array}{ll}a & b \\ c & d\end{array}\right)$ is $D=\left(\operatorname{tr}^{2} M-4\right) / s^{2}$, where $s=\operatorname{gcd}(c, d-a, b)$.

The idea in Duke's Theorem is to look at the equidistribution of closed geodesics on the modular surface, but instead of studying them individually, one may, like classes of binary forms, group them by discriminant. First some examples. 
16

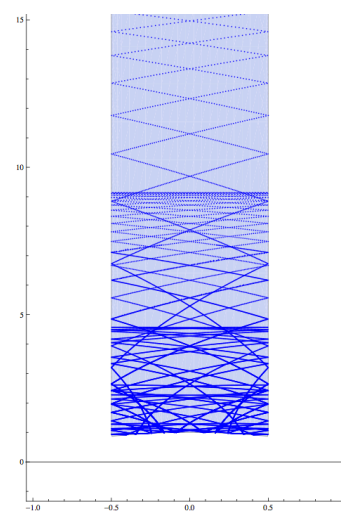

(a) $D=1337$

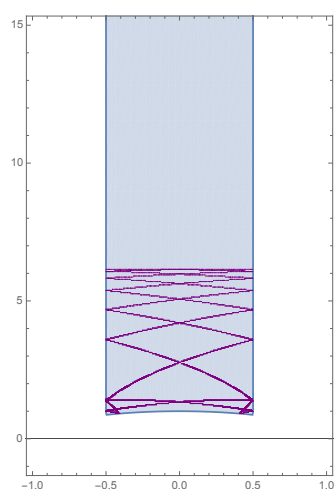

(d) $D=1365: \alpha_{5}, \alpha_{6}$
A. Kontorovich

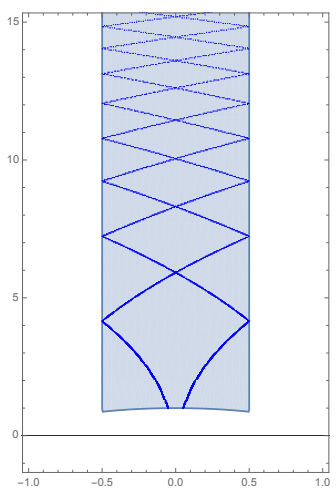

(b) $D=1365: \alpha_{1}, \alpha_{2}$

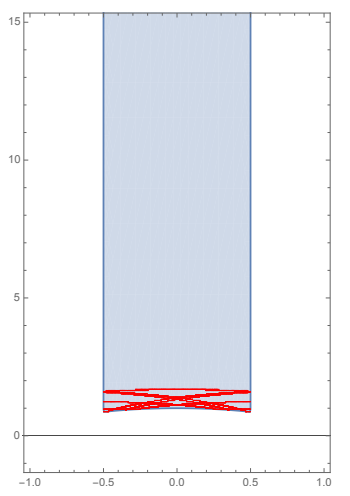

(e) $D=1365: \alpha_{7}, \alpha_{8}$

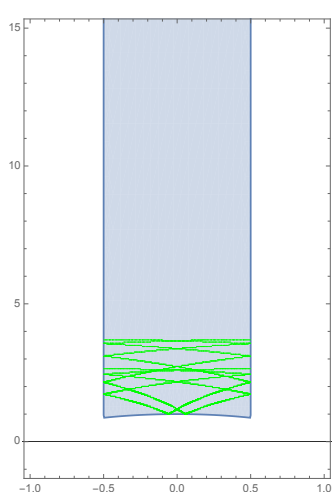

(c) $D=1365: \alpha_{3}, \alpha_{4}$

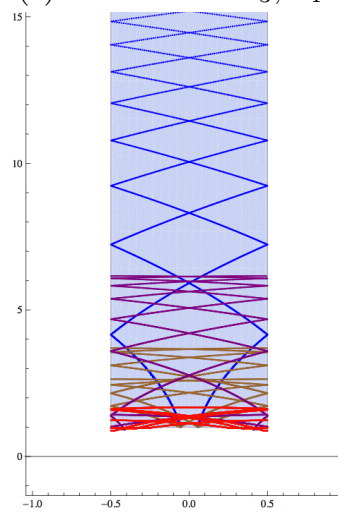

(f) $D=1365:$ All

Figure 1.4 The geodesics in $\mathscr{C}_{D}$ corresponding to (1.33) and (1.34).

For $D=1337$ (which is $\equiv 1(\bmod 4)$ and square-free, and hence fundamental), we find that the class number is $h_{1337}=2$ and the class group $\mathscr{C}_{1337}$ is comprised of two classes, represented by $Q_{1}=[7,-35,-4]$ and $Q_{2}=[4,-35,-7]$. Following the duality above to hyperbolic matrices, we find that $Q_{1}$ and $Q_{2}$ correspond, respectively, to

$$
M_{1}=\left(\begin{array}{cc}
2676336167 & 523561808 \\
299178176 & 58527127
\end{array}\right), \quad \text { and } \quad M_{2}=M_{1}^{\top} .
$$

The entries are so large because the fundamental solution $(T, S)=(t, s)$ to $T^{2}-1337 S^{2}=4$ is:

$$
(t, s)=(2734863294,74794544),
$$


which also explains why the class group is so relatively small (cf. the discussion below (1.27)). The larger roots $\alpha_{1}$ and $\alpha_{2}$ of $Q_{1}(x, 1)$ and $Q_{2}(x, 1)$, respectively, have continued fraction expansions:

$$
\begin{gathered}
\alpha_{1}=[8,1,17,2,1,1,3,1,35,1,3,1,1,2,17,1,8,5] \\
\alpha_{2}=[\overline{5,8,1,17,2,1,1,3,1,35,1,3,1,1,2,17,1,8] .}
\end{gathered}
$$

These have the same even length, and differ by an odd shift (by one), so the two forms $Q_{1}$ and $Q_{2}$ are equivalent under $\mathrm{GL}_{2}(\mathbb{Z})$-action but not under $\mathrm{SL}_{2}(\mathbb{Z})$. That is, while the narrow class number of 1337 is 2 , the full class number is 1 . The corresponding geodesics are actually the same curve, but with opposite orientation; so they look the same in $\Gamma \backslash \mathbb{H}$ but differ in $T^{1}(\Gamma \backslash \mathbb{H})$, because the tangent vectors all change direction. See Figure 1.4a for an illustration, which appears as just one curve, since the tangent vectors are not displayed.

For another example, we study the case $D=1365$. This discriminant is also $\equiv 1(\bmod 4)$ and square-free, and therefore fundamental. This time the class number is $h_{1365}=8$ (Exercise), and we also leave it an exercise to work out representatives for the classes $\left[Q_{1}\right], \ldots,\left[Q_{8}\right]$, the corresponding hyperbolic matrices $M_{1}, \ldots, M_{8}$, and the Pellian solution $(t, s)$. The roots $\alpha_{1}, \ldots, \alpha_{8}$ have continued fractions:

$$
\begin{gathered}
\alpha_{1}=[\overline{1,35}], \alpha_{2}=[\overline{35,1}], \alpha_{3}=[\overline{5,7}], \alpha_{4}=[\overline{7,5}], \\
\alpha_{5}=[\overline{1,1,1,11}], \alpha_{6}=[\overline{1,1,11,1}] \\
\alpha_{7}=[\overline{1,1,1,2,1,2}], \alpha_{8}=[\overline{1,1,2,1,2,1}] .
\end{gathered}
$$

Each pair $\alpha_{2 j-1}, \alpha_{2 j}$ is the same geodesic but with opposite orientation; the four distinct geodesics in $\Gamma \backslash \mathbb{H}$ are illustrated in Figures 1.4b-1.4e. Recall from (1.13) that the cutting sequence of the geodesic flow is a symbolic coding of the visual point; then the first pair of $\alpha$ 's above corresponds to a geodesic that simply shoots up in the air and falls back down (Figure 1.4b), the next two pairs are mid-level geodesics (Figures $1.4 \mathrm{c}$ and $1.4 \mathrm{~d}$ ), and the last pair of $\alpha$ 's gives a very low-lying geodesic (Figure 1.4e).

So the behavior of each geodesic in $\mathscr{C}_{1365}$ is quite different, while there is only one geodesic (up to orientation) in $\mathscr{C}_{1337}$. That said, combining all four geodesics in $\mathscr{C}_{1365}$ into one picture (Figure 1.4f), one finds that, were the geodesics in $\mathscr{C}_{1365}$ all colored the same, it would be quite difficult 
to distinguish this image from Figure 1.4a for $\mathscr{C}_{1337}$. Moreover the density of both plots is reminiscent of the invariant measure $d x d y / y^{2}$ on $\mathbb{H}$ from (1.2). That is, these curves, when grouped by discriminant, become "equidistributed" with respect to the invariant measure as the discriminant grows. Equidistribution here means that the average amount of time this union of curves spends in a given nice region, say $A \subset \Gamma \backslash \mathbb{H}$, becomes proportional to the area of the region. This observation was turned into a beautiful theorem by Duke in [Duk88]. To formulate the statement more precisely, suppose such an $A$ is given, and write $\mathbf{1}_{A}$ for the indicator function of $A$ in $\Gamma \backslash \mathbb{H}$.

Theorem 1.1.2 (Duke's Theorem) As $D \rightarrow+\infty$ through fundamental discriminants,

$$
\frac{1}{h_{D}} \sum_{\gamma \in \mathscr{C}_{D}} \frac{1}{\ell(\gamma)} \int_{\gamma} \mathbf{1}_{A} d s \longrightarrow \frac{1}{\operatorname{vol}(\Gamma \backslash \mathbb{H})} \int_{\Gamma \backslash \mathbb{H}} \mathbf{1}_{A} \frac{d x d y}{y^{2}} .
$$

Here $\ell(\gamma)$ is the length of the closed geodesic $\gamma$, and $d s$ is (hyperbolic) arclength measure.

For simplicity, we have stated (1.35) for the base space $\Gamma \backslash \mathbb{H}$, but a similar result holds for the unit tangent bundle as well. Also, one can prove (1.35) with effective (power savings) rates, and dropping the "fundamental" condition, see, e.g., [CU04]. Again, this is a big theory of which we have only scratched the surface; the proceeding discussion will suffice for our purposes here.

\subsection{Lecture 2: Three Problems in Continued Fractions: ELMV, McMullen, and Zaremba}

\subsubsection{ELMV}

Duke's proof of (1.35) is a tour-de-force of analytic gymnastics, involving a Maass "theta correspondence" to convert period integrals into Fourier coefficients of half-integral weight forms, and implementing methods pioneered by Iwaniec [Iwa87] (using Kuznestov's formula, sums of Kloosterman sums, and estimates of Bessel-type functions) to give non-trivial estimates of such. Meanwhile, the theorem itself (1.35) seems like a beautifully simple dynamical statement; perhaps there is a more "ergodictheoretic" proof? Indeed, one was eventually obtained, after much work, 
by Einsiedler-Lindenstrauss-Michel-Venkatesh [ELMV12] (henceforth referred to as ELMV), with many ideas preempted decades earlier by Linnik [Lin68].

ELMV wanted to approach the problem (and higher rank analogs [ELMV09]) from a type of "measure rigidity" á la Rather's theorems one must show that the only measure arising as a limit of the measures on the left hand side of (1.35) is the Haar measure $d x d y / y^{2}$. This raised the question: does one really need the full average over the class group here, or could individual geodesics already equidistribute? Despite some partial progress to the affirmative (see, e.g., [HM06, Pop06]), because of the symbolic coding of the geodesic flow, closed geodesics can be made to have arbitrary behavior, simply by choosing the partial quotients in the visual point and working backwards. So certainly not all long closed geodesics equidistribute. But perhaps if one restricts only to fundamental closed geodesics, that is, ones whose corresponding discriminant is fundamental, the equidistribution is restored? Even then it is easy to produce examples of non-equidistributing sequences of closed geodesics, which, e.g., have limit measure $d y / y$ supported on the imaginary axis (see [Sar07, p. 233]). But what if we do not allow the "mass" to escape into the cusp of $\Gamma \backslash \mathbb{H}$ ? Can we find closed fundamental geodesics which stay away from the cusp? Among these would certainly be some interesting limiting measures, and would demonstrate the difficulty of Duke's Theorem. Such considerations naturally led ELMV around 2004 to propose the following

Problem: (ELMV) Does there exist a compact subset $\mathcal{Y} \subset \mathcal{X}=$ $T^{1}(\Gamma \backslash \mathbb{H})$ of the unit tangent bundle of the modular surface which contains infinitely many fundamental closed geodesics?

The answer to this question turns out to be YES, as resolved (in a nearly best-possible quantitative sense) by Bourgain and the author in [BK15a]. We will say more about the proof in the last lecture, but first move on to two other (seemingly unrelated) problems.

\subsubsection{McMullen's (Classical) Arithmetic Chaos Conjecture}

Here is a more recent problem posed by McMullen [McM09, McM12], which he calls "Arithmetic Chaos" (not to be confused with Arithmetic 
Quantum Chaos, for which see, e.g. [Sar11].) The problem's statement begins in a similar way to ELMV, asking for closed geodesics on $\mathcal{X}$ contained in a fixed compact set $\mathcal{Y}$, but the source is quite different.

McMullen was studying questions around the theme of Margulis's conjectures on the rigidity of higher-rank torus actions, and observed that a very interesting problem in rank 1 had been overlooked. ${ }^{3}$ The statement is the following.

Conjecture 1.2.1 (Arithmetic Chaos) There is a compact subset $\mathcal{Y}$ of $\mathcal{X}$ such that, for all real quadratic fields $K$, the set of closed geodesics defined over $K$ and lying in $\mathcal{Y}$ has positive entropy.

Before explaining the meaning of the above words, we reformulate the conjecture as a simple statement about continued fractions.

Conjecture 1.2.2 (Arithmetic Chaos II) There is an $A<\infty$ so that, for any real quadratic field $K$, the set

$$
\left\{\left[\overline{a_{0}, a_{1}, \ldots, a_{\ell}}\right] \in K: \text { all } a_{j} \leq A\right\}
$$

has exponential growth (as $\ell \rightarrow \infty$ ).

The reformulation is quite simple. Recall yet again that the geodesic flow is a symbolic coding of the continued fraction expansion of the visual point. Thus going high in the cusp means having large partial quotients, and vice-versa. So a geodesic which is "low-lying" in some compact set $\mathcal{Y}$, that is, avoiding the cusp, is one whose visual point has only small partial quotients. Now since closed geodesics correspond to classes of binary forms with roots that are quadratic irrationals, the visual points automatically lie in some real quadratic field; in fact, it is easy to see that they lie in $K=\mathbb{Q}(\sqrt{D})$, where $D$ is the discriminant of the geodesic (i.e. that of the class). This explains the appearance of real quadratic fields in both versions of the conjecture, as well as how being "lowlying" in Conjecture 1.2.1 corresponds to having small partial quotients in Conjecture 1.2.2. Without defining entropy, let us simply say that this condition in the first version corresponds in the second to the exponential growth of the set in (1.36).

In the third lecture below, we will present a certain "Local-Global Conjecture" which would easily imply McMullen's in the strongest from, that is, with $A=2$ in Conjecture 1.2.2. The same conjecture also has as

3 See [McM09, Conj. 6.1] for a more precise statement which implies Arithmetic Chaos, and moreover predicts that the entropy discussed below can be made arbitrarily close to the natural limit. 
an immediate consequence the aforementioned resolution of the ELMV Problem, as well as Zaremba's Conjecture (to be described below). Unlike the latter two problems, it seems McMullen's problem requires the full force of this Local-Global Conjecture; embarrassingly, the only meager progress made so far is numerical, as we now describe.

In McMullen's lecture [McM12], he gives numerical evidence for Conjecture 1.2 .2 with $A=2$, taking the case $K=\mathbb{Q}(\sqrt{5})$ : he is able to find the following (primitive, modulo cyclic permutations and reversing of partial quotients) continued fractions:

$[\overline{1}]=\frac{1+\sqrt{5}}{2}, \quad[1,1,1,1,1,1,2,1,1,2,2,1,1,1,1,2,2]=\frac{554+421 \sqrt{5}}{923}$.

McMullen presents these two surds as evidence of "exponential" growth.

Using the Local-Global Conjecture as a guide, the author found the following further examples:

$$
\begin{gathered}
{[\overline{1,1,1,2,1,2,2,2,2,1,1,2,2,1,2,1,1,2,2,1}]=\frac{90603+105937 \sqrt{5}}{207538},} \\
{\left[\overline{2,1,1,2,1,1,1,1,2,2,1,1,1,1,1,1,1,1,1,2]}=\frac{12824+7728 \sqrt{5}}{11667} .\right.}
\end{gathered}
$$

Most recently, Laurent Bartholdi and Dylan Thurston (private communication) have pushed these numerics even further, finding the following further examples:

$[\overline{1,1,1,1,1,1,2,2,2,1,1,1,1,1,2,1,1,1,2,2,2,1,2,2,1,1,1,2]}$, $[\overline{1,1,1,1,1,2,2,1,1,1,2,1,1,1,1,1,2,2,2,1,2,1,1,1,1,2,2,2}]$, $[\overline{1,1,1,2,1,1,2,1,2,2,2,2,2,2,1,2,1,1,2,1,2,1,1,2,1,2,2,2,2,2,2,1,2,2}]$, $[\overline{1,1,1,1,1,2,2,1,1,1,2,2,1,2,1,2,2,1,2,2,2,1,1,2,2,2,2,2,1,2,2,1,2,1,2,2}]$, $[\overline{1,1,1,1,2,1,1,2,2,1,1,1,2,2,1,1,1,2,2,2,1,2,1,1,2,2,2,1,1,1,2,1,1,1,2,2}]$, $[\overline{1,1,2,1,2,1,1,2,2,2,1,1,2,1,2,2,2,2,1,1,2,2,1,2,1,1,2,2,2,1,1,2,1,2,2,2}]$, $[\overline{1,1,1,1,1,1,1,1,2,1,1,1,1,1,2,2,2,2,2,2,1,2,2,1,2,2,2,1,1,2,1,2,1,2,2,2,2,1,2,1,1,2]}$, $[\overline{1,1,1,1,1,1,1,1,1,2,1,1,2,1,1,1,1,1,2,1,2,2,2,2,2,1,1,2,1,2,2,2,2,2,1,2,1,2,2,1,2,2,2]}$, $[\overline{1,1,1,2,1,1,2,1,2,2,1,1,1,2,1,2,1,1,2,1,1,1,2,2,1,1,1,2,2,2,2,2,1,1,1,2,2,2,2,1,1,1,2,2}]$. 
all in $\mathbb{Q}(\sqrt{5})$. One may now argue whether this list (of 13 distinct surds in all) is yet indicative of exponential growth.

\subsubsection{Zaremba's Conjecture}

Our final problem originates in questions about pseudorandom numbers and numerical integration. A detailed discussion of these questions is given in $[\mathrm{Kon} 13, \S 2]$, so we will not repeat it here. The statement of the conjecture, understandable by Euclid, is as follows. ${ }^{4}$

Conjecture 1.2.3 (Zaremba [Zar72]) There is some $A<\infty$ such that, for every integer $d \geq 1$, there is a coprime integer $b \in(0, d)$ such that the reduced rational $b / d$ has the (finite) continued fraction expansion

$$
\frac{b}{d}=\left[0, a_{1}, \ldots, a_{\ell}\right]
$$

with all partial quotients $a_{j}$ bounded by $A$.

Progress on the advertised Local-Global Conjecture allowed Bourgain and the author to resolve a density version of Zaremba's Conjecture:

Theorem 1.2.4 ([BK14]) There is an $A<\infty$ such that the proportion of $d<N$ for which Zaremba's conjecture holds approaches 1 as $N \rightarrow \infty$.

In the original paper, $A=50$ was sufficient, and this has since been reduced to $A=5$ in [Hua15, FK14]. Most recently, Zaremba's conjecture has found application to counterexamples to Lusztig's conjecture on modular representations, via the groundbreaking work of Geordie Williamson, see [Wil15].

\subsection{Lecture 3: The Thin Orbits Perspective}

All three problems discussed in Lecture 2 are collected here under a common umbrella as a "Local-Global" problem for certain "thin" orbits. We discuss recent joint work with Jean Bourgain which settles the first problem (ELMV), and makes some progress towards the last (Zaremba).

To motivate the discussion, recall from the Exercise below (1.32) and the expression (1.7) that the quadratic surd

$$
\alpha=\left[\overline{a_{0}, a_{1}, \ldots, a_{\ell}}\right]
$$

\footnotetext{
4 See $[\mathrm{McM} 09, \S 6]$ for McMullen's connection of Zaremba's Conjecture to
} Arithmetic Chaos. 
is fixed by the matrix

$$
M=\left(\begin{array}{cc}
a_{0} & 1 \\
1 & 0
\end{array}\right) \cdot\left(\begin{array}{cc}
a_{1} & 1 \\
1 & 0
\end{array}\right) \cdots\left(\begin{array}{cc}
a_{\ell} & 1 \\
1 & 0
\end{array}\right),
$$

with

$$
\alpha \in \mathbb{Q}\left(\sqrt{\operatorname{tr}^{2} M-4}\right) .
$$

This tells us that studying traces of such matrices $M$, we might learn something about both ELMV and McMullen's conjectures. For example:

Exercise: If $\operatorname{tr}^{2} M-4$ is squarefree, then the corresponding closed geodesic is fundamental.

Yet another elementary exercise is that, if $b / d=\left[0, a_{1}, \ldots, a_{\ell}\right]$, then

$$
\left(\begin{array}{cc}
a_{1} & 1 \\
1 & 0
\end{array}\right) \cdots\left(\begin{array}{cc}
a_{\ell} & 1 \\
1 & 0
\end{array}\right)=\left(\begin{array}{cc}
d & b \\
* & *
\end{array}\right)
$$

so studying the top-left entries of matrices of the above form tells us about Zaremba's conjecture.

In all the above problems, the partial quotients $a_{j}$ are bounded by some absolute constant $A$. Thus we should study the semigroup generated by matrices of the form $\left(\begin{array}{ll}a & 1 \\ 1 & 0\end{array}\right)$, with $a \leq A$. Actually, we will want all elements in $\mathrm{SL}_{2}(\mathbb{Z})$ (whereas the generators have determinant -1 ), so we define the key semigroup

$$
\Gamma_{A}:=\left\langle\left(\begin{array}{ll}
a & 1 \\
1 & 0
\end{array}\right): a \leq A\right\rangle^{+} \cap \mathrm{SL}_{2}
$$

of even length words in the generators; here the superscript + denotes generation as a semigroup.

We first claim that this semigroup $\Gamma_{A}$ is thin. We give the definition by example. As soon as $A \geq 2$, the Zariski closure of $\Gamma_{A}$ is $\mathrm{SL}_{2}$, that is, the zero set of all polynomials vanishing on all of $\Gamma_{A}$, is that of the single polynomial $P(a, b, c, d)=a d-b c-1$. The integer points, $\mathrm{SL}_{2}(\mathbb{Z})$, of the Zariski closure grow like:

$$
\#\left(\mathrm{SL}_{2}(\mathbb{Z}) \cap B_{X}\right) \asymp X^{2},
$$

where $B_{X}$ is a ball about the origin of radius $X$ in one's favorite fixed archimedean norm. On the other hand, an old result of Hensley [Hen89] gives that

$$
\#\left(\Gamma_{A} \cap B_{X}\right) \asymp X^{2 \delta_{A}} .
$$


Here $\delta_{A}$ is the Hausdorff dimension of the "limit set" $\mathfrak{C}_{A}$ of $\Gamma_{A}$, defined as follows:

$$
\mathfrak{C}_{A}:=\left\{\left[0 ; a_{1}, a_{2}, \ldots\right]: \forall j, a_{j} \leq A\right\} .
$$

The dimensions of these Cantor-like sets have been studied for a long time [Goo41, JP01], e.g.,

$$
\delta_{2} \approx 0.5312, \quad \delta_{3} \approx 0.7056, \quad \delta_{4} \approx 0.7889,
$$

and as $A \rightarrow \infty$, Hensley [Hen92] showed that

$$
\delta_{A}=1-\frac{6}{\pi^{2} A}+o\left(\frac{1}{A}\right) .
$$

The point is that all of these dimensions $\delta_{A}$ are strictly less than 1 , so it follows from (1.37) that

$$
\#\left(\Gamma_{A} \cap B_{X}\right)=o\left(\#\left(\mathrm{SL}_{2}(\mathbb{Z}) \cap B_{X}\right)\right),
$$

as $X \rightarrow \infty$. This is the defining feature of a so-called thin integer set (defined this way in [Kon14, p. 954]) - it has archimedean zero density in the integer points of its Zariski closure. When the set in question is actually a subgroup of a linear group, this definition agrees with the "other" definition of thinness, namely the infinitude of a corresponding co-volume, or index; see, e.g., [Sar14].

The ELMV, McMullen and Zaremba problems do not just study the semigroup $\Gamma_{A}$ itself; they all study the image of $\Gamma_{A}$ under some linear map $F$ on $M_{2 \times 2}(\mathbb{Z})$ taking integer values on $\Gamma_{A}$. For example, in Zaremba's conjecture, one takes $F:\left(\begin{array}{ll}a & b \\ c & d\end{array}\right) \mapsto a$ which picks off the top-left entry. For ELMV and Arithmetic Chaos, one studies the trace, $F:\left(\begin{array}{ll}a & b \\ c & d\end{array}\right) \mapsto a+d$. Then the key object of interest is $F\left(\Gamma_{A}\right) \subset \mathbb{Z}$, and even more precisely, the multiplicity with which an integer $n$ is represented in $F\left(\Gamma_{A}\right)$. We define the multiplicity as:

$$
\operatorname{mult}(n):=\#\left\{\gamma \in \Gamma_{A}: F(\gamma)=n\right\}
$$

A priori this count may be infinite (e.g., if $F$ is constant), so it is useful to also define a quantity guaranteed to be finite, by truncating:

$$
\operatorname{mult}_{X}(n):=\#\left\{\gamma \in \Gamma_{A} \cap B_{X}: F(\gamma)=n\right\} .
$$

Since $F$ is linear, the image of an archimedean ball $B_{X}$ will also be of 
order $X$, so one may naively expect the multiplicity of some $n \asymp X$ to be of order

$$
\frac{1}{X} \cdot \#\left(\Gamma_{A} \cap B_{X}\right) \asymp X^{2 \delta_{A}-1} .
$$

It is easy to see that such a prediction is too primitive, e.g., if $F:\left(\begin{array}{ll}a & b \\ c & d\end{array}\right) \mapsto$ $2 a$, then all odd integers are missing in the image.

Given $\Gamma_{A}$ and $F$, we define an integer $n \in \mathbb{Z}$ to be admissible if it passes all "local obstructions":

$$
n \in F\left(\Gamma_{A}\right) \quad(\bmod q),
$$

for every integer $q \geq 1$. While this condition may seem difficult to verify (for instance, it asks about infinitely many congruences!), it turns out that, thanks to the theory of Strong Approximation, it is very easy to check in practice; see, e.g., [Kon13, §2.2] for a discussion.

The Local-Global Conjecture, formulated by Bourgain and the author states that, once these local obstructions are passed, the naive heuristic (1.39) holds.

Conjecture 1.3.1 (The Local-Global Conjecture) Assume that $A \geq$ 2, so that $\Gamma_{A}$ is Zariski dense in $\mathrm{SL}_{2}$, and that its image under the linear map $F$ is infinite; equivalently the Zariski closure of $F\left(\Gamma_{A}\right)$ is the affine line. For a growing parameter $X$ and an integer $n \asymp X$ which is admissible, we have

$$
\operatorname{mult}_{X}(n)=X^{2 \delta_{A}-1-o(1)} .
$$

Notice that the dimensions in (1.38) all exceed 1/2, whence the exponents $2 \delta_{A}-1$ in (1.40) are all positive. So for large $X$, these multiplicities are non-zero, that is, large numbers which pass all local obstructions should be "globally" represented in $F\left(\Gamma_{A}\right)$.

We leave it as a pleasant Exercise to prove McMullen's Conjecture 1.2.2 from (1.40); see also [BK13, Lemma 1.16].

The progress leading to Theorem 1.2.4 uses the fact that the Zaremba map $F:\left(\begin{array}{ll}a & b \\ c & d\end{array}\right) \mapsto a$ is of "bilinear type," in that $F$ can be written as $F(M)=\left\langle v_{1} \cdot M, v_{2}\right\rangle$, where $v_{1}=v_{2}=(1,0)$. A similar result can be proved (see [BK10]) whenever $F$ is of this form; equivalently (Exercise) whenever $\operatorname{det} F=\alpha \delta-\beta \gamma=0$, where $F:\left(\begin{array}{ll}a & b \\ c & d\end{array}\right) \mapsto \alpha a+\beta b+\gamma c+\delta d$.

Quite recently, a more precise formulation of Zaremba's conjecture, based on the Local-Global Conjecture, and the execution of certain Hardy-Littlewood-type estimations and identities for the "singular series" was given by Cohen [Coh15], a Rutgers summer REU student. 


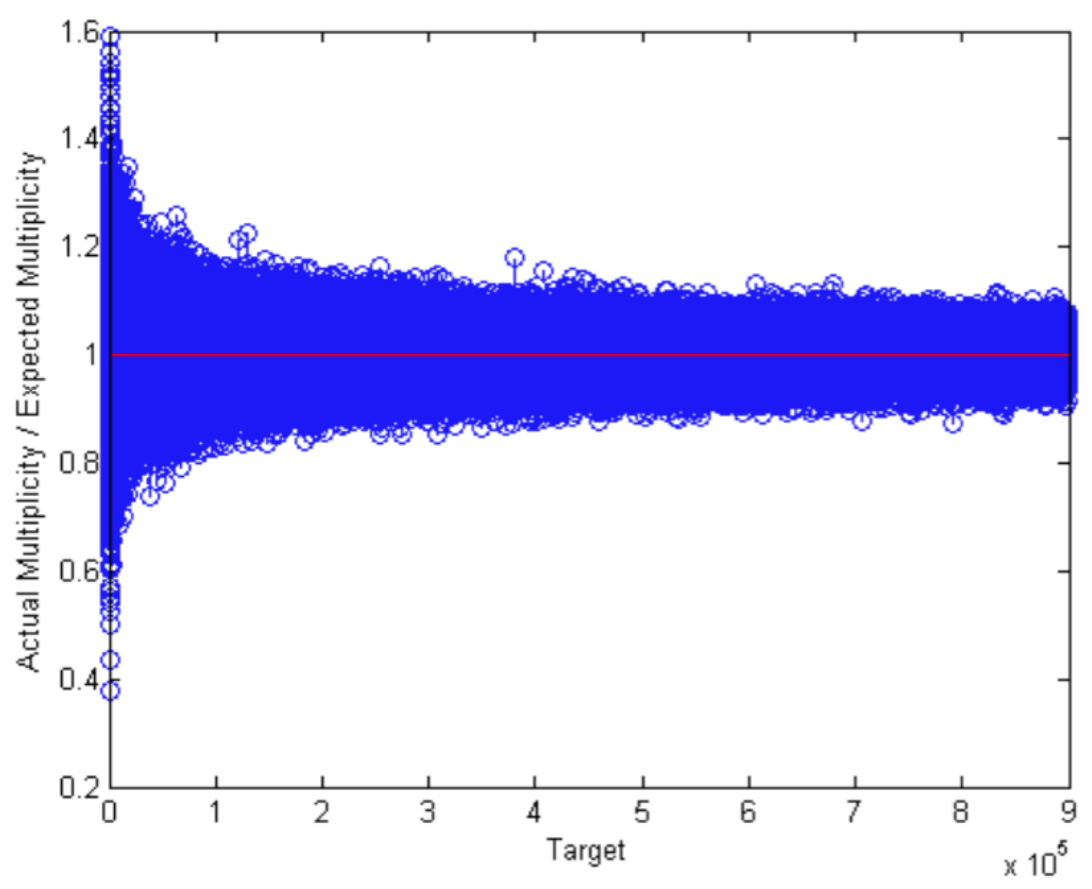

Figure 1.5 Numerical verification of Conjecture 1.3.2 for $A=5$. Image by $\mathrm{P}$. Cohen in [Coh15].

Conjecture 1.3.2 In the Zaremba setting of $F:\left(\begin{array}{ll}a & b \\ c & d\end{array}\right) \mapsto a$, we have

$$
\operatorname{mult}(n) \sim 2 \delta_{A} \frac{\#\left(\Gamma_{A} \cap B_{n}\right)}{n} \times \frac{\pi^{2}}{6} \times \prod_{p \mid n}\left(1-\frac{1}{p}\right) .
$$

A plot of the left hand side divided by the right hand side is given in Figure 1.5; the data is rather convincing in support of the refined conjecture, which hopefully also serves as evidence for the more general Local-Global Conjecture.

\subsubsection{Tools: Expansion and Beyond}

We give here but a hint of some of the methods developed to prove the results in [BK14, BK15a]. A key initial ingredient is what we shall refer to broadly as "expansion," or "SuperApproximation." This has 
also been discussed rather extensively in numerous surveys, see, e.g., [Sar04, Lub12], and [Kon13, §5.2], [Kon14, §3.3]. To go "Beyond Expansion," one needs to develop more sophisticated tools outside the scope of this survey; a hint is given in [Kon14, §3.7], and the interested reader is invited to peruse the original papers [BK15b, BK15a, BK16].

Acknowledgements The author is grateful to the organizers for the opportunity to collect these topics, and to Jean Bourgain for the collaborative work described here. Thanks also to Valentin Blomer and Curt McMullen for comments on an earlier draft.

\section{References}

[BK10] J. Bourgain and A. Kontorovich. On representations of integers in thin subgroups of SL(2, Z). GAFA, 20(5):1144-1174, 2010. 25

[BK13] Jean Bourgain and Alex Kontorovich. Beyond expansion II: Traces of thin semigroups, 2013. Arxiv posting, arXiv:1310.7190. 25

[BK14] J. Bourgain and A. Kontorovich. On Zaremba's conjecture. Annals Math., 180(1):137-196, 2014. 22, 26

[BK15a] J. Bourgain and A. Kontorovich. Beyond expansion II: Low-lying fundamental geodesics, 2015. To appear, JEMS arXiv:1406.1366. $19,26,27$

[BK15b] Jean Bourgain and Alex Kontorovich. The Affine Sieve Beyond Expansion I: Thin Hypotenuses. Int. Math. Res. Not. IMRN, (19):9175-9205, 2015. 27

[BK16] J. Bourgain and A. Kontorovich. Beyond expansion III: Reciprocal geodesics, 2016. Preprint. 27

[BM00] M. Bachir Bekka and Matthias Mayer. Ergodic theory and topological dynamics of group actions on homogeneous spaces, volume 269 of London Mathematical Society Lecture Note Series. Cambridge University Press, Cambridge, 2000. 3

[Cas78] J. W. S. Cassels. Rational Quadratic Forms. Number 13 in London Mathematical Society Monographs. Academic Press, London-New York-San Francisco, 1978. 8, 14

[CL84] H. Cohen and H. W. Lenstra, Jr. Heuristics on class groups of number fields. In Number theory, Noordwijkerhout 1983 (Noordwijkerhout, 1983), volume 1068 of Lecture Notes in Math., pages 33-62. Springer, Berlin, 1984. 13

[Coh15] P. Cohen. A heuristic for multiplicity computation for zarembas conjecture, 2015. Rutgers University Summer 2015 REU paper. 25,26

[Cox13] David A. Cox. Primes of the form $x^{2}+n y^{2}$. Pure and Applied Mathematics (Hoboken). John Wiley \& Sons, Inc., Hoboken, NJ, 
second edition, 2013. Fermat, class field theory, and complex multiplication. 8

[CU04] L. Clozel and E. Ullmo. Equidistribution des pointes de hecke. In Contribution to automorphic forms, geometry and number theory, pages 193-254. Johns Hopkins Univ. Press, 2004. 18

[Dav80] H. Daveport. Multiplicative Number Theory, volume 74 of Grad. Texts Math. Springer-Verlag, New York, 1980. 12

[Deu33] Max Deuring. Imaginäre quadratische Zahlkörper mit der Klassenzahl 1. Math. Z., 37(1):405-415, 1933. 13

[Duk88] W. Duke. Hyperbolic distribution problems and half-integral weight Maass forms. Invent. Math., 92(1):73-90, 1988. 18

[ELMV09] Manfred Einsiedler, Elon Lindenstrauss, Philippe Michel, and Akshay Venkatesh. Distribution of periodic torus orbits on homogeneous spaces. Duke Math. J., 148(1):119-174, 2009. 19

[ELMV12] Manfred Einsiedler, Elon Lindenstrauss, Philippe Michel, and Akshay Venkatesh. The distribution of closed geodesics on the modular surface, and Duke's theorem. LEnseignement Mathematique, 58:249-313, 2012. 19

[EW11] Manfred Einsiedler and Thomas Ward. Ergodic theory with a view towards number theory, volume 259 of Graduate Texts in Mathematics. Springer-Verlag London, Ltd., London, 2011. 3

[FK90] Robert Fricke and Felix Klein. Vorlesungen über die Theorie der elliptischen Modulfunktionen. 2 Bände, Teubner, Leipzig, 1890. 14

[FK14] Dmitrii A. Frolenkov and Igor D. Kan. A strengthening of a theorem of Bourgain-Kontorovich II. Mosc. J. Comb. Number Theory, 4(1):78-117, 2014. 22

[GF51] I. M. Gel'fand and S. V. Fomin. Unitary representations of Lie groups and geodesic flows on surfaces of constant negative curvature. Doklady Akad. Nauk SSSR (N.S.), 76:771-774, 1951. 3

[Gol76] Dorian M. Goldfeld. The class number of quadratic fields and the conjectures of Birch and Swinnerton-Dyer. Ann. Scuola Norm. Sup. Pisa Cl. Sci. (4), 3(4):624-663, 1976. 13

[Goo41] I. J. Good. The fractional dimensional theory of continued fractions. Proc. Cambridge Philos. Soc., 37:199-228, 1941. 24

[GZ86] Benedict H. Gross and Don B. Zagier. Heegner points and derivatives of $L$-series. Invent. Math., 84(2):225-320, 1986. 13

[Hei34] H. Heilbronn. On the class number in imaginary quadratic elds. Quarterly J. of Math., 5:150-160, 1934. 13

[Hej83] Dennis A. Hejhal. The Selberg trace formula for $\operatorname{PSL}(2, \mathbf{R})$. Vol. 2, volume 1001 of Lecture Notes in Mathematics. Springer-Verlag, Berlin, 1983. 14

[Hen89] Doug Hensley. The distribution of badly approximable numbers and continuants with bounded digits. In Théorie des nombres (Quebec, PQ, 1987), pages 371-385. de Gruyter, Berlin, 1989. 23 
[Hen92] Doug Hensley. Continued fraction Cantor sets, Hausdorff dimension, and functional analysis. J. Number Theory, 40(3):336-358, 1992. 24

[HM06] Gergely Harcos and Philippe Michel. The subconvexity problem for Rankin-Selberg $L$-functions and equidistribution of Heegner points. II. Invent. Math., 163(3):581-655, 2006. 19

[Hua15] ShinnYih Huang. An improvement to Zaremba's conjecture. Geom. Funct. Anal., 25(3):860-914, 2015. 22

[Hum16] G. Humbert. Sur les fractions continues ordinaires et les formes quadratiques binaires indéfinies. Journal de mathématiques pures et appliquées 7e série, 2:104-154, 1916. 7

[IK04] Henryk Iwaniec and Emmanuel Kowalski. Analytic number theory, volume 53 of American Mathematical Society Colloquium Publications. American Mathematical Society, Providence, RI, 2004. 12, 14

[Iwa87] H. Iwaniec. Fourier coefficients of modular forms of half-integral weight. Invent. Math., 87:385-401, 1987. 18

[JP01] Oliver Jenkinson and Mark Pollicott. Computing the dimension of dynamically defined sets: $E_{2}$ and bounded continued fractions. Ergodic Theory Dynam. Systems, 21(5):1429-1445, 2001. 24

[Kon13] Alex Kontorovich. From Apollonius to Zaremba: local-global phenomena in thin orbits. Bull. Amer. Math. Soc. (N.S.), 50(2):187228, 2013. 22, 25, 27

[Kon14] Alex Kontorovich. Levels of distribution and the affine sieve. Ann. Fac. Sci. Toulouse Math. (6), 23(5):933-966, 2014. 24, 27

[Lag80] J. C. Lagarias. On the computational complexity of determining the solvability or unsolvability of the equation $x^{2}-d y^{2}=-1$. Trans. AMS, 260(2):485-508, 1980. 13

[Lan35] E. Landau. Bemerkungen zum Heilbronnschen Satz. Acta Arith, pages 1-18, 1935. 13

[Lin68] Yu. V. Linnik. Ergodic properties of algebraic fields. Translated from the Russian by M. S. Keane. Ergebnisse der Mathematik und ihrer Grenzgebiete, Band 45. Springer-Verlag, New York, NY, 1968. 19

[Lub12] A. Lubotzky. Expander graphs in pure and applied mathematics. Bull. Amer. Math. Soc., 49:113-162, 2012. 27

[McM09] Curtis T. McMullen. Uniformly Diophantine numbers in a fixed real quadratic field. Compos. Math., 145(4):827-844, 2009. 19, 20, 22

[McM12] C. McMullen. Dynamics of units and packing constants of ideals, 2012. Online lecture notes, http://www.math.harvard.edu/ ctm/ expositions/home/text/papers/cf/slides/slides.pdf. 19, 21

[Pop06] A. Popa. Central values of Rankin $L$-series over real quadratic fields. Compos. Math., 142:811-866, 2006. 19

[Sar80] Peter Clive Sarnak. PRIME GEODESIC THEOREMS. ProQuest 
LLC, Ann Arbor, MI, 1980. Thesis (Ph.D.)-Stanford University. 14

[Sar04] P. Sarnak. What is...an expander? Notices Amer. Math. Soc., 51(7):762-763, 2004. 27

[Sar07] Peter Sarnak. Reciprocal geodesics. In Analytic number theory, volume 7 of Clay Math. Proc., pages 217-237. Amer. Math. Soc., Providence, RI, 2007. 14, 19

[Sar11] Peter Sarnak. Recent progress on the quantum unique ergodicity conjecture. Bull. Amer. Math. Soc. (N.S.), 48(2):211-228, 2011. 20

[Sar14] Peter Sarnak. Notes on thin matrix groups. In Thin Groups and Superstrong Approximation, volume 61 of Mathematical Sciences Research Institute Publications, pages 343-362. Cambridge University Press, 2014. 24

[Ser85] Caroline Series. The modular surface and continued fractions. $J$. London Math. Soc. (2), 31(1):69-80, 1985. 8

[Sie35] Carl Ludwig Siegel. Über die analytische Theorie der quadratischen Formen. Ann. of Math. (2), 36(3):527-606, 1935. 13

[Wil15] G. Williamson. Schubert calculus and torsion explosion, 2015. arXiv:1309.5055v2 with Appendix by A. Kontorovich, P. McNamara, G. Williamson. 22

[Zar72] S. K. Zaremba. La méthode des "bons treillis" pour le calcul des intégrales multiples. In Applications of number theory to numerical analysis (Proc. Sympos., Univ. Montreal, Montreal, Que., 1971), pages 39-119. Academic Press, New York, 1972. 22 